\title{
José Martí, Paulo Freire y Hugo Zemelman: la tecnología desde una educación emancipadora
}

José Martí, Paulo Freire and Hugo Zemelman: Technology based upon a Emancipatory Education

José Martí, Paulo Freire e Hugo Zemelman:

Tecnologia desde uma Educação

Emancipadora

Joao Gabriel Rodrigues Telles-Almeida* iD orcid.org/0000-0002-5176-2289

Para citar este artículo: Telles-Almeida, J. (2O21). José Martí, Paulo Freire y Hugo Zemelman: la tecnología desde unaeducaciónemancipadora.RevistaColombianadeEducación,/(81),249-268.https://doi.org/10.17227/rce.num8110924

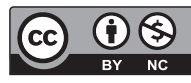

Recibido: 11/12/2019

Evaluado: 19/09/2020

* Candidato a Doctor en comunicación por la Universidad Pompeu Fabra. Instituto de Pensamiento y 


\section{Resumen}

Este artículo encuentra en tres pensadores fundantes de la epistemología crítica en educación claves para la constitución de la categoría educación popular transmedia para pensar la educación con una comunidad específica ante sus prácticas tecnológicas con énfasis en las digitales. Se suman lecturas de autores contemporáneos, como Jorge Huergo, Raúl Mejía, Estela Quintar y Tania Pérez Bustos, lo que redunda en propuestas didácticas y consecuencias políticas de la articulación entre las provocaciones de investigadores y la práctica del autor como educador. Algunos de los aportes fundamentales son la idea del valor del diálogo como contraposición a la imposición de la masividad, pensar la biografía tecnológica como dispositivo didáctico, el valor del proyecto como eje de la construcción de propuestas educativas que involucren la tecnología, la incorporación del aprendizaje informal como parte de un proceso situado y pensar la tecnología como un eje transversal en lugar de una disciplina.

\section{Palabras clave}

educación tecnológica; tecnología de la información; educación popular; comunicación aumentativa y alternativa; usos de tecnología en educación; pensamiento crítico

\section{Keywords}

technology education; information technology; popular education; augmentative and alternative communication; technology uses in education; critical thinking

\begin{abstract}
This article shows key concepts in three main thinkers of the critical epistemology in education to propose the category of transmedia popular education. This construct makes it possible to think of education with a specific community in terms of their technological practices, particularly the digital ones. We also discuss this topic with contemporary authors such as Jorge Huergo, Raúl Mejía, Estela Quintar and Tania Pérez Bustos, creating didactical tools and political consequences arising from the articulation between the researchers ideas and the author's practice as an educator. Some of the fundamental contributions are dialogue as a category to critique massive education, technological biography as a didactical tool, the value of the project as the axis of the construction of educational proposals that involve

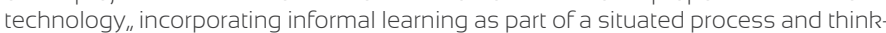
ing about technology as a cross-curricular axis rather than as a particular discipline.
\end{abstract}

\section{Resumo}

Este artigo encontra em três pensadores fundantes da epistemologia crítica na educação ideias centrais para a constituição da categoria educação popular transmedia como uma categoria para pensar a educação com uma comunidade específica ante suas práticas tecnológicas com ênfase nas digitais, aliando com isso leitura de autores e autoras contemporâneas como Jorge Huergo, Raúl Mejía, Estela Quintar e Tania Pérez Bustos para resultar em propostas didáticas e consequências políticas da articulação entre as provocações de investigadores e a prática do autor como educador. Alguns dos aportes fundamentais são pensar o valor do diálogo como contraposição à imposição da massividade, pensar a biografia tecnológica como um dispositivo didático, o valor do projeto como eixo de construção de propostas educativas que incorporem a tecnologia, a incorporação da aprendizagem informal como parte de um processo situado e pensar a tecnologia como um eixo transversal e não como uma disciplina.

\section{Palavras-chave}

educação tecnológica; tecnologia da informação; educação popular; comunicação aumentativa e alternativa; usos da tecnologia na educação; pensamento crítico 


\section{Introducción: educación popular como hija de cambios técnicos}

Las prácticas y reflexiones que culminaron en el famoso libro de Paulo Freire Pedagogía del oprimido (1987) vinieron de una demanda creciente de reinserción laboral de masas de migrantes campesinos a la ciudad a finales de los años cincuenta y principios de los sesenta. Esto obligaba una formación básica de esa cantidad de trabajadores analfabetas para incorporarse a la entonces joven industrialización brasilera. Una relación muy similar a lo que hoy nos vemos obligados a reflexionar: un cambio técnico radical que implica nuevas coordenadas de lógicas de trabajo y estructuras cognitivas que eliminan formas de sociabilidad y empleabilidad, en las cuales se vive la posibilidad de convertir diferencias generacionales y geográficas en abismos sociales. Así como en los cincuenta se vivenció un gran cambio social que obligó a los sectores comprometidos con las transformaciones políticas y sociales a definir una epistemología para responder a las tendencias excluyentes o de puro adiestramiento para el trabajo impuestas por el modelo económico vigente, necesitamos redefinir coordenadas para hacer la misma apuesta en el marco de los cambios globales generados por la creciente digitalización de la vida humana.

Este artículo propone rescatar tres referentes del pensamiento crítico en educación: Paulo Freire, José Martí y Hugo Zemelman, para, a través de sus contribuciones epistémicas, definir marcos metodológicos de lo que venimos proponiendo como educación popular transmedia en respuesta al desafío de época propuesto.

\section{Cuestión preliminar: de qué mundo hablamos o la disputa entre Monsanto y los bancos de semillas ${ }^{1}$}

Muchos autores intentan definir los cambios generados por las nuevas tecnologías. Me referiré a tres puntos relevantes para este trabajo:

»El capitalismo no sobrevive más de vender mercancías, ahora alquila conocimiento. No hablamos más de un capitalismo industrial clásico que explota a un trabajador y se queda con el excedente de su trabajo. Se trata de industrias cada vez más robotizadas y un número cada vez más pequeño de operarios, lo que realmente garantiza la ganancia de grandes empresas son sus grandes laboratorios

1 Este apartado de contextualización es rescatado por el autor del artículo "Un posible proyecto de educación popular transmedia" (Almeida, 2019), en el cual se relata la experiencia del primer proyecto educacional que fundamentó la escritura de este artículo. 
de ingenieros, diseñadores, programadores, entre otros profesionales capaces de incorporar el máximo posible de conocimiento a sus mercancías, asesorados por equipos de abogados y lobistas que garantizan la propriedad privada de esos desarrollos y un intento creciente de diferenciarse en el más mínimo detalle para acceder a nichos de consumidores (Blondeau et al., 2004).

McLuhan (1962) ya había señalado en los años sesenta que la tecnología es la extensión del cuerpo humano. Eso se vuelve cada vez más vigente, pues nuestra vivencia en las ciudades pasa a ser coordinada por las aplicaciones de geolocalización que utilizamos, nuestros gustos y criterios de sociabilidad empiezan a ser reducidos a burbujas de amistades y gustos definidos por algoritmos de redes sociales, y nuestros mismos criterios de verdad son establecidos por las fuentes de información, las cuales legitiman al grupo social al que pertenecemos a través de métricas de interacciones. Incluso los regresos idílicos a una vida analógica, como los de los compradores de vinilos, las ecoaldeas, entre otras tendencias, son mediadas por recibir interacciones positivas en redes sociales. Como bien describe Eric Sadin (2018), vivimos una silicolonización del mundo, en la cual reforzamos identidades y guetos sociales a través de las respuestas positivas y negativas que nuestra existencia en redes sociales nos proporciona.

» Hay un hiato jurídico. El mundo, en particular la lógica de los Estados-nacionales, no está preparado para la vida social en redes, y no sabe muy bien cómo reaccionar a una nueva juventud que sufre más influencias de su tribu transmedia que de las instituciones tradicionales, como partidos, iglesias o escuelas.

Todos estos puntos comparten un mismo problema de fondo: la propiedad. Interpretando lo que Zemelman (2006) definía como horizontes de posibilidades del momento histórico, los tres puntos, vistos de otro modo, podrían ofrecer un mundo más interesante para vivir. Podríamos pensar en una sociedad en la cual el ser humano estaría libre de trabajos que obligan a que hoy el $85 \%$ de los trabajadores mundiales estén insatisfechos, de acuerdo con la encuesta de Gallup ("Sólo el $15 \%$ de los trabajadores se sienten comprometidos con sus empleos", 2018). También nos permitiría pensar en la utilización de los códigos y algoritmos para el uso racional de recursos, o para un sistema de participación política más efectivo, incluso sistemas de circulación de mercancía en cadenas locales que contribuyan a disminuir la huella de carbono en el mundo, sumando a eso la posibilidad de pensar modos de intercambio más baratos y directos entre comunidades, a fin de extinguir fronteras nacionales y favorecer la cooperación entre los pueblos. 
Sin embargo, con el creciente monopolio de las tecnologías en manos de grandes empresas, vemos todo lo contrario: Eliminación de puestos de trabajo sin perspectiva para quienes quedan desempleados, lo que obliga a flujos migratorios que fortalecen el discurso de las fronteras nacionales, y el ordenamiento social definido por grandes propiedades privadas que buscan maneras cada vez más eficientes de detentar el máximo de tecnología disponible.

Un ejemplo de nuestro contexto que puede definir la contradicción del momento es la disputa entre el modelo de banco de semillas y Monsanto. La manipulación genética es tan antigua como la agricultura. Desde que el ser humano empezó a cultivar, buscó identificar sus mejores plantas para fortalecer la producción. Eso ha permitido que las comunidades tengan una diversidad gigante de semillas que representan el cúmulo de siglos y de generaciones que a través de un intercambio horizontal han podido generar una manipulación sostenible de la genética para favorecer a sus comunidades. La globalización ha facilitado, en el marco de los movimientos sociales agrarios, en particular del Sur Global, intercambios tecnológicos transnacionales y gratuitos. Ese es el paradigma de otro mundo posible cuando la tecnología tiene una propiedad comunal. En el otro extremo está el modelo Monsanto. ("Los 'monstruos' de Monsanto: más de un siglo envenenando el planeta, 2015").

Más allá de la discusión de los impactos en la salud, un punto relevante sobre los transgénicos es que son semillas generadas en laboratorio para tener perfecta adaptabilidad a un modelo de cultivo determinado: el monocultivo transnacional, para sobrevivir al uso masivo de pesticidas y otros venenos que son necesarios para ese tipo de producción. Y lo más importante, esas semillas son patentadas por el laboratorio que las desarrolla. Siendo así, con inversión masiva y apoyo de Estados nacionales, Monsanto vende el paquete completo de semillas y agrotóxicos, que van cambiando el mismo ecosistema de los países y volviéndolos dependientes de su tecnología de manera más o menos voluntaria, pues al contrario de la crianza de animales que puede ser mejor controlada, la forma de procreación de una planta es mucho más caótica y diversa ya que en ella interfieren factores como el viento, animales dispersores, entre otros. De manera que, si por casualidad una planta cultivada desde una semilla transgénica llega a cruzarse con otro tipo, debido a la lógica de propiedad privada del conocimiento, los agricultores son obligados a pagarles por el uso de las semillas contaminadas por la modificación genética. La disputa entre modelos de agricultura nos permite visualizar de modo mucho más intuitivo el punto central de los dos modelos de sociedad que realmente están en juego: o una sociedad fundada en la alienación tecnológica de grandes masas de población que dependen de la voluntad y proyectos 
económicos de grandes monopolios, o una sociedad en la cual las comunidades detentan la soberanía tecnológica consciente y definen su forma de vida a través de su uso.

\section{José Martí: los maestros ambulantes 2.0}

En un texto de 1884, José Martí introdujo el concepto de maestros ambulantes como alternativa para la educación en el campo. Su premisa es que muchas regiones alejadas de América Latina no tendrían otra manera de acceder a la educación si no fuera a través de estrategias en las cuales los maestros fueran de pueblo en pueblo enseñando:

Esa idea de los "maestros ambulantes" es acaso la única solución práctica del problema de la enseñanza en los países de mucho campo, o de poblaciones de pocos habitantes. El maestro tiene que ir a aquellos que no pueden ir al maestro. Y como la técnica es pesada y poco gustosa, no se debe ser, ni en el campo ni en la ciudad, ni en la escuela fija ni en la escuela a caballo, maestro de técnica, sino de práctica. No deben enseñarse reglas sino resultados. (Martí, 1884).

Ese texto trae dos lecturas potenciales del presente histórico. La primera y más evidente es la relación con el espacio. Rescatando la noción de extensión de McLuhan citada, podemos pensar las redes sociales como el nuevo caballo de los maestros ambulantes. En lugar de la lógica colonizadora de los cursos abiertos masivos en línea (MOоc) $)^{2}$ que utiliza la enseñanza virtual para imponer desde la educación bancaria una visión de mundo fundamentada en autores anglosajones (Altbach, 2014), el uso de los espacios virtuales para una enseñanza popular en América Latina debe servir para superar la problemática concreta de generar encuentros entre personas distanciadas espacialmente. La cuestión la pensó de manera profunda en América Latina Darcy Ribeiro (1995), quien ya había percibido el potencial de democratización y superación de las desigualdades regionales a través de la enseñanza virtual, inspirado en la Open University de Londres y entendiendo a su vez que existía el riesgo de que eso se transformara en una "máquina de hacer dinero".

Pero más allá del aspecto de democratización regional, hay otra enseñanza de Martí. En el texto citado, el gran pensador cubado nos dijo que para la "religión nueva" serían necesarios "sacerdotes nuevos". La idea del maestro que se monta en el caballo y viaja hasta los rincones de la patria grande propone una ética distinta para pensar la formación de los

2 Abreviatura en inglés de Massive Open Online Courses, iniciativa de cursos universitarios abiertos y masivos promovido principalmente por universidades de Estados Unidos y Reino Unido a través de plataformas como EdX. 
educadores, la cual es potencializada con la internet. Significa pensar que, en lugar de poner en manos de los educandos la tarea de acceder a los espacios de aprendizaje, los proyectos de educación emancipadora tienen que buscar una manera, parafraseando la famosa canción de Milton Nascimento, de estar donde el pueblo está. El uso de la internet para eso significa una innegable reducción de costos y la ventaja de promover encuentros de miembros de las patrias chicas (Ramos, 2010) en una perspectiva de construcción de un saber para la patria grande. Obviamente las diferencias de acceso siguen siendo un problema, pero es necesario destacar que entre el 2010 y el 2015 América Latina ya presentó un aumento de suscripciones móviles en $802,5 \%$ y de conexiones fijas en $68,9 \%$ " 5 datos sobre el acceso a internet en América Latina, 2016". Estamos hablando de una tendencia hasta el momento creciente de la conectividad en la región, con énfasis en la juventud.

La segunda contribución de Martí es la vigencia que tiene su idea de que el maestro ambulante es a la vez un maestro práctico. Como dijimos, la disputa que se vuelve cada vez más urgente pasa por la soberanía tecnológica (Hache, 2018), en un sentido paralelo a aquel en el que los movimientos agrarios en América Latina plantean la soberanía alimentaria, es decir, pensando prácticas colectivizadas de propiedad y sentido del uso de las tecnologías. Los nuevos sujetos sociales tienen los artefactos cada vez más integrados a sus cuerpos, somos cada vez más cíborgs (Haraway). La frontera entre lo digital y lo analógico son cada vez más inexistentes. Así, perder la soberanía sobre la tecnología es incluso perderla sobre nuestros cuerpos. Utilizando de nuevo la metáfora de Monsanto, así como la semilla genéticamente modificada hace que el cultivo de los campesinos sea propiedad de esta empresa, los códigos de programación informática que ordenan nuestra vida, una vez producidos por fuera de nuestras necesidades colectivas, permiten que nuestra experiencia del mundo, digitalmente mediada, sea propiedad de empresas como Google o Facebook. Y las lógicas contemporáneas de enseñanza "inclusiva" en este sentido no cambian ese paradigma.

Pensemos en el famoso ejemplo de Laboratoria (https://www.laboratoria.la/). Fundada en 2014, se trata de una iniciativa creada en México para formar mujeres de bajos ingresos con el objetivo de que puedan trabajar en el mercado de la tecnología, en el cual hoy solo un $10 \%$ de los programadores son mujeres. A pesar de ser una iniciativa bastante positiva en términos de ofrecer perspectivas laborales a personas históricamente excluidas de nuestra sociedad está apoyada, entre otros, por Google, Microsoft y el BID. Es decir, si bien permite que sectores marginalizados aprendan cómo programar, ese saber no se queda en las comunidades a las que estas mujeres pertenecen, simplemente es una estrategia para integrarlas como trabajadoras de los grandes monopolios tecnológicos, 
con sueldos proporcionalmente más bajos que los de los programadores de Estados Unidos y Europa, lo que favorece los márgenes de ganancia de dichos monopolios.

En este aspecto, el pensamiento de José Martí tiene una vigencia radical. Es necesario pensar cómo, desde un saber práctico, se puede construir esa soberanía tecnológica en clave comunitaria. Así como la educación popular tiene su origen en la noción de que la construcción de conciencia viene aliada a la demanda concreta de enseñar a leer y escribir, necesitamos pensar el código de programación, los microcontroladores, el modelaje y la impresión 3D y el manejo de imagen en ambientes digitales, como saberes prácticos en los cuales la construcción de conciencia es un aliado. Necesitamos reaprender de Paulo Freire.

\section{Paulo Freire: diálogo, conciencia y nuestro Zuckerberg hospedado}

Paulo Freire es un autor del cual podemos decir que carga la misma maldición que el Che Guevara. Como bien expone el documental Personal Che (2007), esos personajes sobrepasaron fronteras ideológicas y generacionales, y su imagen ha sido adaptada al gusto de quien la consuma. El Paulo Freire que se quiere rescatar es el militante en el marco de la reforma agraria chilena, en especial, el que escribe los libros Pedagogía del oprimido en 1968 y Extensão ou Comunicação? de $1969 .{ }^{3}$ La elección de estos dos textos se fundamenta en el análisis histórico que presentamos en la introducción de este artículo. Nos interesa de manera destacada el Paulo Freire que tenía la tarea concreta de pensar un modelo educativo vinculado a los cambios económicos de su época. Son tres las contribuciones de estos textos al debate aquí propuesto:

» Incluso cuando hablamos de un mundo mediado por la internet, la función central de la educación popular sigue siendo permitir al ser humano formarse una conciencia de su relación con la realidad en que está implicado a través de la problematización de esa realidad (Freire, 1983). En el contexto del uso de las nuevas tecnologías, eso significa, entre otras cosas, romper con la idea de los algoritmos como una caja negra (Bucher, 2016), reflexionar acerca de los cambios en el mercado promovidos por las marcas de amor (Jenkins, 2006), licencias y usos colectivos de la tecnología, como los softwares de código abierto (Open Source Initiative) y los derechos de uso Creative Commons. Al contrario de iniciativas direccionadas a la "inclusión" de sectores marginados a los

3 En términos de citación utilizaremos, respectivamente, las versiones brasileras de Paz e Terra, 1987 y 1983. 
mercados, justificadas bajo la ilusión de pequeñas Silicon Valley distribuidas en el mundo (Sadin, 2018), debemos pensar cómo reflexionar acerca de la tecnología en función de las realidades concretas de los territorios.

» Es necesario destacar aquí la crítica que hace Paulo Freire a la invasión cultural. Como bien destaca el educador brasilero, y después Kaplún (2002) intentará exponer en detalle, el intento persuasivo es per se una acción colonizadora y adversa a la educación popular, pues intenta negar las experiencias concretas del Otro en nombre de una idea pensada como solución para todos los problemas. Debemos romper tanto el optimismo tecnológico, que muchas veces ofrece la entrada alienada al mercado de los datos, como la visión apocalíptica que niega a las comunidades la construcción de una relación con la tecnología. Rescatando otra metáfora de Paulo Freire (1987), es necesario pensar el conectivo con en lugar de para como clave del entendimiento de la tecnología y, en consecuencia, la comunicación. La soberanía tecnológica no es el resultado de pensar una tecnología para el pueblo, como es actualmente la propuesta de los estudios de mercado y audiencia, pero sí de pensarla con el pueblo, vinculándolos como sujetos activos de la construcción de esos saberes ubicados en sus realidades concretas.

»El diálogo (Freire, 1987, 1983) como categoría es el concepto central que diferencia una metodología emancipadora de las demás. Independientemente de si son análogos o digitales, los espacios de educación crítica tienen que fundamentarse en el debate, la mediación de ideas y síntesis de formulaciones colectivas. Los espacios expositivos tienen que ser secundarios, y no el centro del espacio de enseñanza.

Por un lado, la utilización empírica de las redes sociales como forma de aprendizaje en plataformas tales como YouTube, Code Wars o Udemy, tienen su fundamento en la práctica como eje de aprendizaje a través de tutoriales y comunidades de foros que, a su vez, sirven de soporte para la construcción de esos saberes. Al hacerse sin mediación didáctico-crítica, es difícil que esos saberes sobrepasen la función de preparación para el mercado o como pasatiempo, dos funciones legítimas, pero que no es el enfoque que proponemos. Cuando, a su vez, pensamos los usos de la tecnología en propuestas de educación alternativa, tomando como ejemplo los cursos virtuales de Clacso, percibimos que no hay una reflexión profunda acerca de la relación con la tecnología, que es la propia materialidad del proceso educativo. Las clases son exposiciones del profesor encargado, con foros que deberían reproducir 
la idea presentada, pero que, en lugar de promover el diálogo y la construcción colectiva de saberes, sirve de espacio para subir tareas propuestas por los profesores.

El conocimiento técnico, en particular de herramientas de código abierto, está considerablemente diseminado en redes digitales, y cualquiera con tiempo y saberes para transitar en foros de usuarios lo puede aprender. Pero, si lo que se quiere es generar conciencia desde ese aprendizaje, necesitamos rescatar el diálogo alrededor del uso de esas técnicas, romper el carácter mecanicista de aprender-aplicar. Es necesario destacar que no estamos acá proponiendo reinventar la rueda. Sabemos que hay iniciativas locales, en su gran mayoría presenciales y analógicas, que vienen pensando en ello. El Nordeste brasilero ha sido un campo fecundo de iniciativas en ese sentido, por ejemplo la red Baobáxia. Posiblemente hay otras y diversas experiencias locales en América Latina que no conocemos. Pero el diferencial de lo que proponemos es que, así como la lectoescritura tiene que ser pensada en la totalidad de los procesos de enseñanza, la tecnología también lo tiene que ser, pues es parte de la nueva existencia social, y aquellos que no sean capaces de vivirla serán excluidos como históricamente lo han sido quienes no saben leer o escribir.

Por esta razón utilizamos como ejemplo los seminarios Clacso, que como no están vinculados a esa perspectiva, reproducen la idea de que se puede enseñar pensamiento crítico en plataformas digitales sin reflexionar alrededor del uso de las tecnologías. En consecuencia, el diálogo, que es el corazón de una propuesta de educación popular, en nuestra interpretación, se pierde en ese tipo de seminario, porque no hay razonamiento y construcción lo suficientemente profundos sobre cómo y cuáles son las consecuencias en términos de número de estudiantes, contenido y dispositivos didácticos. Sin pensar esas cuestiones, no será un proceso de educación emancipadora sino un intento de persuadir el pensamiento de los profesores de los seminarios.

Un término acuñado en Pedagogía del oprimido, que parece un tanto olvidado, es el de opresor hospedado. En resumen, Paulo Freire nos recuerda que muchos de los campesinos se comportan tal cual como sus opresores cuando son ascendidos a capataces (Freire, 1987). Quienes aprenden a convivir en redes sociales, a hacer videos, editar imágenes, entre otros saberes, se vuelven los representantes legítimos para posicionar la imagen de sus colectivos, comunidades o identidades en la esfera pública. Existen riesgos de que, entre otras cosas, los que aprendan esos saberes, en general jóvenes, se ilusionen con el ascenso económico que pueden significar 
esos conocimientos, en especial la programación. También pueden emerger lógicas cada vez menos colectivas de utilización tecnológica, dejando para los "más listos" la función de cuidar de este aspecto. Volviendo a las metáforas agrícolas, Freire propone una solución: no pensar desde el "pesticida", o sea, eliminar los potenciales causantes del daño, sino desde una lógica de permacultura ("¿Qué es permacultura?", s. f.) Hay que pensar cómo integrar a los jóvenes que son señalados, en general, como los más interesados en la tecnología en el ecosistema de sus comunidades, de manera que puedan confrontar el "Zuckerberg" hospedado en muchos jóvenes de esa generación. Nos referimos al fundador de Facebook, y la ilusión muy presente y analizada, por ejemplo por Sadin (2018), de que todo joven en el capitalismo cognitivo es un millonario desarrollador de aplicaciones en potencia.

\section{Zemelman: el discurso ante la tecnología}

Rescatamos la charla "El futuro como ciencia y utopía" dada en la UNAM y el libro El conocimiento como desafío posible, en la edición de 2006 realizada por el Instituto de Pensamiento y Cultura en América Latina, para pensar la contribución de Hugo Zemelman a ese debate. Las elegimos por el realce que da el autor a la tecnología y a la educación en esos trabajos.

Una cuestión que podemos afirmar de antemano es que la visión de Zemelman sobre la tecnología era de desconfianza. Sus preocupaciones esenciales eran la temporalidad de la construcción del conocimiento, la exclusión de intelectuales que no estaban "insertados" en las lógicas digitales y una carencia de lenguaje producida por el uso de tecnologías (Zemelman, 2006). Sin embargo, siendo coherente con su propia propuesta metodológica, esas reflexiones parecen dialogar con el tiempo histórico de principio de los 2000, en el cual efectivamente había un optimismo de la cibercultura (Lévy, 2009). Si bien son alertas que siguen vigentes, principalmente si miramos los discursos oficialistas en educación, como la creación por parte del IIPE, la Unesco, el Unicef y la Alianza Mundial para la Educación de cursos masivos para "enseñar" cómo deben enseñar los profesores ("Un mundo preparado para aprender", 2019), proponemos que la potencia de su propuesta reside en cuatro afirmaciones categóricas que deben orientar nuestro planteamiento:

» Una afirmación al final de la charla citada sirve de complemento a Paulo Freire para definir la idea fuerza de lo que es la educación popular transmedia. Haciendo un juego de palabras con conectivos, Zemelman dice que la tarea es pensar un pensamiento crítico ante la tecnología, y no sobre. Si miramos un diccionario sobre 
el uso de la preposición ante, percibimos que es un sustituto de en presencia de (Castellano actual, 2012). Al contrario de sobre, que implica un objeto pasivo en lo cual yo produzco una acción, el ante supone que el "objeto" modifica mi accionar. Siendo así, podemos decir que la educación popular transmedia es una categoría para pensar la educación con una comunidad específica ante sus prácticas tecnológicas con énfasis en las digitales, pensando la categoría Transmedia desde Scolari (2018) como la más interesante para definir el conjunto de prácticas implicadas en la vivencia activa en comunidades híbridas virtuales/analógicas.

» Reflexionando sobre la filosofía, el pensador chileno hace una crítica a la enseñanza de esta como una disciplina especializada en la escuela (Zemelman, 2006). De acuerdo con él, la escolarización de la filosofía termina con ella en el ambiente escolar, ya que ese saber humano debería permear todo el conocimiento. Podemos trasladar la misma reflexión a la cuestión de la tecnología. La episteme alrededor de la tecnología y su uso - entendiendo episteme acá como parte de la experiencia, como comportamiento acompañado de pensamiento en función de las acciones diarias de los seres humanos- debe ser tarea de todos los implicados en ambientes educativos, pues cada día se hace difícil imaginar experiencias educacionales sin mediación de dispositivos digitales. Retomando la idea fuerza propuesta, cada comunidad tiene que producir saberes alrededor de sus prácticas transmedias.

»El concepto de proyecto o utopía es esencial para lo que proponemos aquí. Por otro lado, este planteamiento podría ser criticado, de manera razonable, argumentando que si una comunidad no tiene prácticas transmedias esa propuesta es irrelevante, o que la tecnología ha servido hegemónicamente para la dominación. Una de las principales enseñanzas de la epistemología crítica zemelmaniana es que todo presente contiene un futuro en potencial, y que la tarea de una investigación no es describir en presente, sino colocarse ante él y construir esa utopía. Sabemos que las prácticas transmedias están fundadas en la colonialidad del saber (Quijano, 1993), y son estimuladas por una lógica de mercado que nos quiere convertir, día tras día, en datos para vendernos productos. Sin embargo, pensamos que la alternativa a eso no es el boicot, como sugiere Sadin, por ejemplo. Nuestra apuesta es: traer los debates y creaciones ante prácticas transmedias al centro de la labor educacional porque es la única forma de romper ese dispositivo colonial y poner la técnica en razón de una utopía emancipadora. 


\section{Diálogo con autores contemporáneos sobre el mismo tema}

No somos los primeros en pensar una relación entre educación popular, medios y tecnología. Si bien hasta acá nuestro planteamiento se hace a nivel de tecnología, por limitantes de capacidad del diálogo del mismo autor, trataremos con autores que piensan esa relación con las tecnologías de información y comunicación, campo de experiencia de este.

Desde una óptica cuantitativa, Paula Jimena Rodríguez Marino y su equipo (2018) buscaron entender las temáticas abordadas en cincuenta artículos de la base Redalyc sobre "Educación", "Tecnología" y "Comunicación popular" (Rodríguez Marino et al., 2018) utilizando la herramienta QDMiner. Un estudio similar más amplio ya había sido realizado en Brasil, entre 1997 y 2007, con 130 artículos usando la misma herramienta (Peixoto y Araújo dos Santos, 2007).

Joana Peixoto y Claúdia Helena dos Santos Araújo (2007) encontraron que Paulo Freire era el segundo autor más citado en ese campo; ${ }_{4}^{4}$ la gran mayoría de los artículos remitía al autor brasileño para defender el papel de la autonomía del estudiante en el espacio de aprendizaje y la necesidad de abordar la tecnología de manera crítica. El trabajo de Paula Jimena Rodríguez Marino y su equipo también trae esos dos puntos como los centrales en estudios alrededor de ese tema. Desde nuestra interpretación, eso permite decir que ubicarnos dentro del sintagma educación popular no habla más de la enseñanza de un tipo de sujeto histórico específico ("el pueblo"), sino como una manera de ubicarnos en una tradición intelectual que tiene Paulo Freire como punto de partida del diálogo y que intenta contribuir al campo de los estudios críticos a través del entendimiento de la participación activa de las personas implicadas en el proceso educativo. Un punto relevante presentado por el equipo de Rodríguez Marino, que ya comentamos en nuestro análisis de Zemelman, es la implicación de la tecnología en proyectos. Podemos ubicar la comunicación popular, en particular toda la tradición de radios comunitarias, como un paradigma para pensar la educación de tecnología basada en proyectos. Construir un contenido que efectivamente va a ser transmitido, que está dentro de un proyecto comunitario, moviliza el deseo por el saber técnico. La presencia masiva de redes sociales y la diseminación de los celulares inteligentes reducen mucho los costos y ofrecen más condiciones para realizar proyectos concretos en situaciones en las que los sujetos tienen algo que decir. Las conclusiones compartidas con esas autoras vienen del mismo campo experiencial en comunicación.

4 El primero fue Pierri Levy, ya que su famoso texto sobre cibercultura había sido publicado en Brasil por esa época. 
Por otra parte, en la contribución del debate desde el diálogo entre lo comunicativo y educacional, es muy importante rescatar las contribuciones de Jorge Huergo $(2001,2007)$. El primer punto relevante para nuestra propuesta es pensar la comunicación como puesta en común, esto es, pensar que comunicar y dialogar es establecer un encuentro entre discursos en una esfera pública, que va a implicar conflicto y contradicciones. Acá, concordando con el autor, no imaginamos la categoría diálogo en una ilusión de armonía e integración. Si queremos romper al opresor que vive en nosotros mismos, debemos vivir la contradicción del encuentro. Podemos decir que Huergo nos ayuda a precisar la colaboración de Paulo Freire en ese sentido.

El segundo es, posiblemente, ser uno de los primeros en hacer una crítica directa a enseñar una "técnica" fuera del contexto. Tal vez una de sus principales contribuciones al campo es legitimar que la elección del cómo comunicar y las herramientas correspondientes dependen del proceso de realización. Eso quiere decir, por ejemplo, que aprender un editor de video es el resultado de la decisión de hacer un video, no lo contrario. Podemos acá dialogar con las potencialidades descubiertas por Scolari y el grupo Medium (2018) sobre el aprendizaje informal y los medios. Una vez definido el sentido colectivo del qué hacer con la tecnología, los distintos saberes vinculados pueden fácilmente aprenderse de la inteligencia colectiva desde tutoriales, para a su vez resignificar esos saberes en actividades concretas en favor de su comunidad.

El tercer punto es pensar la clave biográfica tecnológica, en sintonía con lo que pensamos desde Ipecal en didactobiografía (Quintar, 2008). En ese punto también se destaca una de las contribuciones de las pedagogías feministas a la educación popular (Pérez Bustos, 2010).

Tania Pérez Bustos nos demuestra cómo los movimientos feministas nos enseñan la necesidad de ubicar las singularidades de los sujetos para romper la ilusión de una ciencia y tecnología no implicada y generalista, como si los criterios de género, raza y clase de quien la produce no fuera relevante. Establece un diálogo con Huergo (2001) al poner las categorías de diferencia, autoridad y experiencia (Pérez Bustos, 2010) como eje central para pensar la labor en el ámbito de la popularización de la tecnología. Una contribución relevante desde esta posición es romper la ilusión del "científico" como un personaje sin errores. Asumir los fracasos, los errores, la experimentación como parte de biografía sobre la tecnología es una consecuencia de los aportes del feminismo a nuestro campo. La reflexión alrededor de la autoridad, al preguntar quién disemina el conocimiento y qué conocimiento, nos ayuda en nuestros cuestionamientos sobre los MOOC. Estela Quintar (2018), sin hablar directamente de tecnología, es una constante de esas reflexiones, pues desde su comprensión de la diversidad y el papel de la colocación del sujeto para la construcción de un proceso crítico es una dialogante de todo lo que venimos formulando. 
Por último, es necesario resaltar que mucho del análisis coyuntural del papel de la tecnología y la definición de premisas éticas desde la educación popular ya fueron materia de reflexión por parte de Marco Raúl Mejía (2004, 2007), de manera muy similar a cómo lo hacemos acá. Creemos que nuestra contribución es permitir una mirada desde lo didáctico y de la experiencia misma de la labor con tecnologías para los avances que Mejía ya había iniciado desde su relación con distintas iniciativas de educación popular en Colombia.

\section{Origen colectivo de las reflexiones}

Antes que nada, es necesario decir que esta no es una propuesta que sale de la cabeza de un individuo. Lo planteado acá es resultado de una trayectoria de media década realizando procesos de enseñanza y aprendizaje alrededor de estos temas, es gracias a algunos de ellos en particular que cabe destacar. El primero surgió al enseñar saberes transmedia a estudiantes del proyecto Pés na Estrada do Conhecimento, gracias a la invitación de la profesora Lisiane Vandressen. Aquel fue el primer momento en el que efectivamente esas problemáticas surgieron para el investigador.

El segundo se dio a través de los cursos de comunicación en el marco del proceso de reincorporación de las FARC-EP en Colombia, de los cuales el investigador participó como profesor de periodismo. La posibilidad de respirar esa temática, durante cuatro meses, junto a una comunidad política organizada permitió el surgimiento de distintas ideas que se ven reflejadas en este texto. Cabe resaltar las contribuciones singulares de Boris Guevara, Julián Caballero, Santiago, Clara y Jacinto, los cuales, en las jornadas de desvelo a lo largo de ese proceso, permitieron conversaciones alrededor de diversos temas, así como a NC Producciones, en particular Manuel Bolívar, que, con su demanda de pensar un curso situado en la problemática específica de la paz, instó al investigador a reflexionar respecto de estas y otras cuestiones. También es importante hacer un reconocimiento a Anderson Pérez, el Monito, estudiante del curso y quien fue violentamente asesinado en el marco de la reincorporación en el 2018. Su inquietud y su curiosidad fueron grandes impulsos para la formulación de esas problemáticas, y agradecemos toda la enseñanza que nos ha ofrecido durante el tiempo que tuvimos el honor de convivir a su lado.

El tercer momento, el actual, se da con la participación del investigador en el Instituto Pensamiento y Cultura en América Latina-Ipecal, gracias a la oferta generosa de Estela Quintar para desarrollar investigaciones relacionadas con la educación popular transmedia en dicha institución. Mucho de lo expuesto acá es resultado de los debates en el marco del seminario "Fake News, Capitalismo cognitivo y Sociedad del consumo", coordinado por el investigador, en el cual participaron los profesores 
e investigadores Lorena Aguirre Rodríguez, Ernestina Benites, Said Abat Jiménez Mayorga, Blanca Irene Valdivia Salas, Cecilia Sánchez Martínez y Horacio Vences Sánchez. Otro espacio fundamental se ha dado a través de la formulación de un proyecto de Ipecal en asociación con el Gitidic, grupo de investigación de la Universidad Nacional de Colombia: el diálogo con Nelson Andrés Ravelo, Laura Alejandra Villamil, Daniel Leonardo Caro

y Christian David Rodríguez ha nutrido el desarrollo de la investigación

\section{Conclusión: guía metodológica para la educación popular transmedia}

Consideramos que sería incoherente con esta propuesta terminar sin elementos didácticos para la discusión. El diálogo de los autores a lo largo de este texto giró no solo alrededor del qué hacer, sino también del cómo hacer. En consecuencia, proponemos:

» Explorar en comunidades de aprendizaje u organizaciones sociales qué prácticas transmediales ya existen y construir intercambio y saber desde ellas.

» Utilizar la biografía del sujeto y su relación con los medios y la tecnología como parte del proceso educativo.

» Crear círculos de reflexión alrededor del uso de la tecnología en comunidades de aprendizaje u organizaciones sociales. El diálogo estimulado por un tema generador (Freire, 1987), elemento metodológico básico de la propuesta freireana, sigue siendo una práctica vigente para situar una reflexión crítica y construcción de conocimiento sobre la tecnología.

» Crear proyectos de educación virtual o a distancia coherentes con la ética implicada. Pensamos en esos proyectos académicos como una derivación de los maestros ambulantes, no como una "máquina de hacer dinero" (Ribeiro, 1995). Para eso, problemáticas como un límite de estudiantes en clase, el carácter del profesor como dinamizador y no como fuente del saber, entre otras conclusiones, ya son lugares comunes en la literatura sobre educación para pensar la viabilidad de modelos educativos no bancarios, que siguen vigentes y tienen que ser tenidos en consideración cuando se hacen proyectos en plataformas digitales. No porque la web permita la masificación tenemos que operar bajo esa lógica.

» Crear y fortalecer los bancos de herramientas Open Source y Creative Commons, así como elaborar material didáctico propio. Es necesario sistematizar y crear dispositivos didácticos en función de lo que se propone en este artículo. Mucho del proceso técnico puede ser hecho desde el "cacharreo" (por ensayo y error/o de manera 
artesanal) con la tecnología, siempre y cuando haya información disponible y los recursos necesarios. Dejar la parte técnica del aprendizaje funcionar de manera más autónoma, principalmente en la elección de las herramientas, permite poner la individualidad del sujeto en el marco de un proyecto colectivo.

Aprendizaje fundamentado en una utopía colectiva. Una de las claves de lo que estamos proponiendo es que se construyan proyectos colectivos que den sentido social a las técnicas en una realidad específica. Los estudios ya comprueban que un joven con buen acceso y tiempo libre puede aprender distintas prácticas en internet, desde saberes propiamente tecnológicos hasta cómo cocinar, maquillarse, etc. ("Transmedia literacy. Exploiting transmedia skills and informal learning strategies to improve formal education"). Ahora, para hacer una creación que no sea calcar/reproducir las lógicas sociales dominantes, o que eso sea posible en espacios excluidos de ese buen acceso, es necesario crear sentido social y reflexiones como comunidad para salir de la lógica mecanicista e individualizada. En este sentido, si el proyecto es colectivo o comunitario, esto permite que aquel que guste de trabajar con fotografía o video pueda dedicarse a aprender eso, lo mismo quien quiera aprender a programar porque encuentra sentido en ello. Pero cada una de esas prácticas ubicadas en un proyecto colectivo ganan sentido y reflexión desde el lugar de lo personal/individual.

Es necesario destacar que ser consecuente con estas conclusiones tiene implicaciones políticas. Hay dos puntos de partida. El primero es posicionar la idea de que contar con tiempo libre de convivencia con aparatos tecnológicos es parte del aprendizaje. La disputa del tiempo para que los miembros de una comunidad puedan ver un tutorial, experimentar con un software, sacar fotos e incluso navegar por redes sociales para entender sus usos son esenciales. La incorporación de ese tiempo como parte del proceso educativo, que implica buscar alternativas económicas para que los implicados tengan ese tiempo y acceso a los artefactos tecnológicos, que permanezcan en la comunidad para aquellos que no tienen como acceder a ellos individualmente, es una de las luchas para la implementación de una propuesta educativa en esa clave. Incluso, es necesario romper la noción de etapas de formación (Huergo, 2011). Estar siempre aprendiendo, y en función de lo que "toca hacer" se presenta como un modelo no lineal y necesario de validar.

El segundo tiene que ver con la elección de los primeros espacios para implementar esa propuesta. En nuestra lectura, debe ser en espacios de formación de formadores. Sea en espacio de formación de liderazgos de movimientos sociales o en cursos universitarios de 
pedagogía y licenciaturas, consideramos que el primer paso es diseñar posibilidades junto a quien podrá mantener a largo plazo esa disputa como multiplicadores.

\section{Referencias}

Almeida, J. G. (2019). Un posible proyecto de educación popular transmedia. Sobre Tudo, 10(2), 225-241.

Altbach, P. G. (2014). Neocolonialismo en los MOoc: ¿quién controla el conocimiento? International Higher Education, 75, 6-8.

Blondeau, O., Corsani, A., Dyer-Whiteford, N., Kyrou, A., Lazarrato, M., Mouler-Boutang, Y., Rullani, E. y Varcellone, C. (2004) Capitalismo cognitivo, propiedad intelectual y creación colectiva. https://www.traficantes.net/sites/default/files/pdfs/Capitalismo\%20cognitivo-Tds.pdf

Bucher, T. (2016). Neither black nor box: Ways of knowing algorithms. En S. Kubitschko y A. Kaun (eds.), Innovative Methods in Media and Communication Research (pp. 81-98). Palgrave Macmillan

Castellano actual. http://udep.edu.pe/castellanoactual/la-preposicion-ante/

"5 datos sobre el acceso a internet en América Latina". (2016, 13 de septiembre). El Economista. https://www.eleconomista.com.mx/ empresas/5-datos-sobre-el-acceso-a-internet-en-America-Latina-20160913-0141.html

Freire, P. (1983). Extensão ou Comunicação? Paz e Terra.

Freire, P. (1987). Pedagogia do oprimido. Paz e Terra.

El futuro como ciencia y utopía [Video] .https://www.youtube.com/watch?$\mathrm{v}=8 \mathrm{hKbd0VUCLk}$

Hache, A. (2018). Soberanía tecnológica. Soberanía Tecnológica (vol. 1). https://archive.org/details/soberaniatecnologica1/page/n3

Haraway, D. (1991). A Cyborg Manifesto: Science, Technology, and Socialist-Feminism in the Late Twentieth Century. En Simians, Cyborgs, and Women: The reinvention of nature (pp. 149-181). Routledge.

https://baobaxia.mocambos.net

https://www.codewars.com

https://creativecommons.org/

https://www.clacso.org/formacion/seminarios-virtuales/

Huergo, J. (2001). La popularización de la ciencia y la tecnología: interpelaciones desde la comunicación [ponencia]. Seminario Latinoamericano Estrategias para la Formación de Popularizadores en Ciencia y Tecnología. Red-Pop- Cono Sur. La Plata, 14 al 17 de mayo de 2001.

Huergo, J. (2007). Los medios y tecnologías en educación. Ministerio de Educación, Ciencia y Tecnología. Dirección Nacional de Gestión 
Curricular y Formación Docente. Unidad de Tecnologías de la Comunicación y la Información. http://repositorio.educacion.gov.ar:8080/ dspace/bitstream/handle/123456789/95679/medios_tecnologias_ huergo.pdf?sequence $=1$

Jenkins, H. (2006). Convergence Culture, la cultura de la convergencia em los medios de comunicación. Paidós Ibérica.

Kaplún, M. (2002). Una pedagogía de la comunicación. Editorial Caminos. Lévy, P. (2009). Cibercultura (trad. Carlos Irineu da Costa). Editora 34.

Martí, J. (1884). Maestros ambulantes. La América Nueva York. (tomo 8). p. 288-292. http://www.josemarti.info/documentos/maestros_ambulantes.html

Mejía, M. R. (2004). La tecnología, la(s) cultura(s) tecnológica(s) y la educación popular en tiempos de globalización. Polis, 2(7), 0-34. http:// www.redalyc.org/articulo.oa?id=30500707

Mejía, M. R. (2007). Tecnología, globalización y reconstrucción de la educación popular. Pasos (Segunda época), 130, 31-42.

McLuhan, M. (1962). The Gutenberg galaxy. University of Toronto Press.

Los "monstruos" de Monsanto: más de un siglo envenenando el planeta. (2015). https://actualidad.rt.com/actualidad/172768-biotecnologia-omg-monsanto-transgenicos

Un mundo preparado para aprender: Llamamiento conjunto en pro de una educación de calidad en la primera infancia para todos. (2019). https://es.unesco.org/news/mundo-preparado-aprender-Ilamamiento-conjunto-pro-educacion-calidad-primera-infancia-todos

Open Source Initiative. https://opensource.org/

Peixoto, J. y Araújo dos Santos, C. H. (2012). Tecnologia e educação: algumas considerações sobre o discurso pedagógico contemporâneo. Educação \& Sociedade, 33(118), 253-268.

Pérez Bustos, T. (2010). Aportes feministas a la Educación popular: entradas para repensar pedagógicamente la popularización de la ciencia y la tecnología. Educação e Pesquisa, 36(1), 243-260.

Personal Che. [Documental]. https://www.youtube.com/watch?v=oxyJDs6rHv8

¿Qué es permacultura? (s. f.). https://www.permacultura.org.mx/es/permacultura/que-es/

Quintar, E. (2008). Didáctica no parametral: un sendero hacia la esperanza. Instituto de Pensamiento y Cultura en América Latina.

Quintar, E. (2018). La diversidad cultural en América Latina. Un problema ético político para la formación y la producción de conocimiento. Paulo Freire. Revista de Pedagogía Crítica,16(19), 221-248.

Ramos, J. A. (2010). Historia de la Nación latinoamericana. Biblioteca Federal. 
Ribeiro, D. (1995). O Brasil como Problema: a formação e o sentido do Brasil. Companhia das Letras.

Rodríguez Marino, N. y Aguirre, V. (2018). Educación, tecnologías y comunicación popular, comunitaria y alternativa. Una revisión cartográfica-conceptual en revistas latinoamericanas. Algarrobo-meL7, 1-16. revistas.uncu.edu.ar

Sadin, E. (2018). La silocolonización del mundo. La irresistible expansión del liberalismo digital. Caja Negra Editora.

Scolari, C. (org.) (2018). Adolescentes, medios de comunicación y culturas colaborativas. Aprovechando las competencias transmedia de los jóvenes en aula. Ce.Ge

Scolari, C. (s. f.). Lo aprendí en un tutorial. http://revistaanfibia.com/ensayo/lo-aprendi-en-un-tutorial/

Scolari, C. (s. f.). Transmedia literacy. Exploiting transmedia skills and informal learning strategies to improve formal education. https:// transmedialiteracy.org/

Solo el 15\% de los trabajadores se sienten comprometidos con sus empleos. (s. f.). https://cepymenews.es/solo-15-por-ciento-trabajadores-se-sienten-comprometiddos-con-sus-empleos/

Zemelman, H. (2006). El conocimiento como desafío posible. Ipecal. 